\title{
Chromogenic in-situ hybridization: a viable alternative to fluorescence in-situ hybridization in the HER2 testing algorithm
}

\author{
Wedad M Hanna and Kevin Kwok \\ Sunnybrook and Women's College Health Sciences Centre, Toronto, ON, Canada
}

\begin{abstract}
Assessment of human epidermal growth factor receptor-2 status is standard practice in women with breast cancer. Most laboratories use immunohistochemistry as a screening test, with equivocal results confirmed by fluorescence in-situ hybridization (FISH). Chromogenic in-situ hybridization (CISH) is a relatively new method for detection of gene amplification using a peroxidase reaction, which can be viewed using a standard light microscope. This study was undertaken to validate $\mathrm{CISH}$ as a method for assessing human epidermal growth factor receptor-2 gene amplification. The gene amplification status of human epidermal growth factor receptor-2 immunohistochemistry negative $(0 / 1+, n=69$; Group 1), immunohistochemistry positive (3+, $n=50$; Group 2$)$ and equivocal tumor samples $(2+, n=135$; Group 3$)$ was evaluated by FISH and $\mathrm{CISH}$, and the concordance between FISH and $\mathrm{CISH}$ results calculated. In Group 1, 67/69 cases did not show amplification by $\mathrm{CISH}$ and 69/ 69 showed no amplification by FISH. Two cases were discordant; therefore, fluorescence/CISH concordance was $97 \%$. In Group 2, 46/50 cases were amplified by FISH and 47/50 cases were amplified by CISH; three cases were not amplified by either method (immunohistochemistry false-positives). Only one case showed discordant FISH and $\mathrm{CISH}$ results, making the fluorescence/CISH concordance 98\%. In Group 3, 89/135 cases were not amplified and $37 / 135$ were amplified by both methods. Nine cases were discordant, giving a fluorescence/CISH concordance of $93 \%$. The discordant cases were those with very low or borderline amplification with FISH. The high level of concordance between FISH and CISH seen in this study suggests that CISH may be a viable alternative to FISH for use in the human epidermal growth factor receptor-2 testing algorithm.
\end{abstract}

Modern Pathology (2006) 19, 481-487. doi:10.1038/modpathol.3800555; published online 27 January 2006

Keywords: HER2 testing; CISH; FISH; concordance; HER2 testing algorithm

The human epidermal growth factor receptor-2 (HER2) is overexpressed in approximately 20-30\% of breast cancers. ${ }^{1,2}$ Overexpression of the HER2 protein has been shown to correlate with amplification of the HER2 gene and is associated with an aggressive disease course and a poor prognosis. ${ }^{3,4}$ HER2 overexpression is also a strong predictor of response to the humanized monoclonal antibody trastuzumab (Herceptin ${ }^{\mathbb{R}}$ ), which has demonstrated a significant survival benefit in patients with HER2-positive breast cancer. ${ }^{5,6}$ Assessment of HER2 status has therefore become standard practice in women with breast cancer in order to identify those patients most likely to benefit from treatment with trastuzumab.

Correspondence: Dr WM Hanna, MB, BCh, FRCPC, Department of Anatomic Pathology, Sunnybrook and Women's College Health Sciences Centre, 2075 Bayview Avenue, Toronto, ON, Canada M4N 3M5.

E-mail: Wedad.Hanna@sw.ca

Received 28 September 2005; revised and accepted 19 December 2005; published online 27 January 2006
Most laboratories in North America and Europe use immunohistochemistry to determine HER2 status, with equivocal results confirmed by fluorescence in-situ hybridization (FISH). Immunohistochemistry identifies HER2 protein expression on the cell surface, while FISH determines the actual degree of HER2 gene amplification. ${ }^{7}$ Both immunohistochemistry and FISH are highly specific and reproducible HER2 testing methodologies when performed using standardized and validated protocols. ${ }^{7}$ Indeed, both methods are recommended in the HER2 testing algorithm, which has been designed to ensure optimal identification of patients eligible for treatment with trastuzumab. ${ }^{8}$ Patients with tumors that are immunohistochemistry $3+$ or FISH positive are considered HER2 positive and are therefore eligible for treatment with trastuzumab, while patients with immunohistochemistry $2+$ tumors should be assessed for amplification of the HER2 gene by FISH to determine their eligibility for therapy. However, both immunohistochemistry and FISH have certain limitations. ${ }^{2}$ While immunohistochemistry is readily available in most laboratories, 
results are susceptible to variations in methodology and operator interpretation between laboratories. FISH is a highly sensitive and specific method for determination of HER2 gene amplification. However, this method is more costly than immunohistochemistry, requiring special skills and expensive equipment, which is not widely available in diagnostic pathology laboratories. Furthermore, assessment of tumor morphology is less than optimal with FISH, making it difficult to distinguish between invasive carcinoma and carcinoma in situ. FISH is also limited by the fact that the fluorescent signal decays rapidly at room temperature, making it difficult to preserve slides for future reference.

Alternative methods of assessing HER2 gene amplification status are therefore being investigated. Chromogenic in-situ hybridization (CISH) is a relatively new method for determination of gene amplification using a peroxidase-based chromogenic reaction, which can be viewed using a conventional bright field microscope. Like FISH, CISH determines the actual degree of HER2 gene amplification. However, unlike FISH, positive signals can be identified using standard laboratory equipment. Histopathology of the specimen can be assessed simultaneously and the signal does not decay, but remains stable over a long period of time, allowing slides to be stored at room temperature. As such, CISH has a wider potential for integration into diagnostic laboratories than FISH. However, as with any new test, technical and clinical validation is required before CISH becomes accepted as standard practice. This study was undertaken to validate CISH as a method for assessing HER2 gene amplification in positive, negative and borderline immunohistochemistry-tested cases. The results were compared with FISH testing carried out on the same tumor samples.

\section{Methods}

\section{Study Design}

CISH and FISH were used to determine the HER2 gene amplification status of the following invasive breast carcinoma samples: HER2 immunohistochemistry-negative $(0,1+)$ tumor samples (Group 1); HER2 immunohistochemistry-positive $(3+)$ tumor samples (Group 2) and tumor samples of equivocal $(2+)$ HER2 immunohistochemical status (Group 3). For each group, the concordance between FISH and CISH results was calculated.

\section{Assays}

All assays were performed according to the manufacturers' recommended protocol. Immunohistochemistry testing was performed using the Hercept Test $^{\mathrm{TM}}$ (DakoCytomation). Two-color FISH analysis was performed using the PathVysion ${ }^{\mathrm{TM}}$ kit (Vysis,
Abbott). CISH testing was performed using the Sunnybrook and Women's College Health Sciences Centre (SWCHSC) modified CISH protocol for HER2. Slides were deparaffinized with xylene and hydrated through $100 \%$ ethanol and graded alcohols to distilled water. Slides then underwent heat pretreatment. The slides were added to a $1 \times$ Antigen decloaker pretreatment solution (Biocare Inc.) in a Coplin Jar and put in an electric pressure cooker (Biocare Inc.) at $120^{\circ} \mathrm{C}$ for $2.5 \mathrm{~min}$. They were then allowed to cool to approximately $75^{\circ} \mathrm{C}$ in the solution, which took approximately $25 \mathrm{~min}$ from peak temperature. The slides were then transferred to Tris buffer at room temperature and treated with Zymed Digest-All III Pepsin solution for 0-3 min. The digestion time was dependent on the time the slides had been fixed in formalin. Following this, the slides were washed with distilled water and dehydrated in graded alcohols and dry air before undergoing co-denaturation and hybridization (using Visys Hybrite incubator). HER2 probes $(10 \mu \mathrm{l})$ were added to the slides and coverslip slides, then sealed with rubber cement. Slides were then denatured at $73^{\circ} \mathrm{C}$ for $10 \mathrm{~min}$, and hybridized at $37^{\circ} \mathrm{C}$ overnight. Following a post-hybridization wash, the rubber cement and coverslip were removed. The slides were then immersed in $2 \times$ saline sodium citrate (SSC) with $0.3 \% \mathrm{NP} 40$ at $72{ }^{\circ} \mathrm{C}$ for 2 min and washed with Tris buffer saline (TBS). Immunodetection was then carried out using Zymed Spot-Light ${ }^{\mathrm{TM}}$ CISH Polymer Detection Kit.

\section{Scoring of Results}

In all cases, scoring was performed by the investigators. No prior knowledge of immunohistochemistry/FISH status was known when scoring CISH. For FISH, chromosome 17 centromeric signals (CEP17; green) and HER2 gene-specific signals (red) were counted within 60 nuclei of invasive cancer cells and the ratio of red/green signals calculated. HER2/CEP17 ratios $<2$ were defined as negative for amplification, with HER2/CEP17 ratios $\geq 2$ defined as positive for amplification. For CISH, the number of HER2 signals per nucleus was counted, with $<6$ dots defined as no amplification and $\geq 6$ as positive amplification.

\section{Results}

A total of 254 tumor samples were scored. Of these, 69 were HER2 negative (immunohistochemistry 0/1+; Group 1), 50 were HER2 positive (immunohistochemistry 3+; Group 2) and 135 were of equivocal HER2/neu status (immunohistochemistry $2+$; Group 3). Concordance results between immunohistochemistry and FISH/CISH are summarized in Table 1. In Group 1, concordance between immunohistochemistry/FISH was 100 and $97 \%$ between immunohistochemistry/CISH (Figure 1). In Group 2, 
Table 1 Immunohistochemistry/FISH and immunohistochemistry/CISH concordance

\begin{tabular}{lccc}
\hline Group & Immunohistochemistry result & FISH concordance (\%) & CISH concordance (\%) \\
\hline 1 & $0 / 1+(n=69)$ & $69 / 69(100)$ & $67 / 69(97)$ \\
2 & $3+(n=50)$ & $46 / 50(92)$ & $47 / 50(94)$ \\
3 & $2+(n=135)$ & $37 / 135$ (positive) & $37 / 135$ (positive) \\
& & $89 / 135$ (negative) & $89 / 135$ (negative) \\
\hline
\end{tabular}
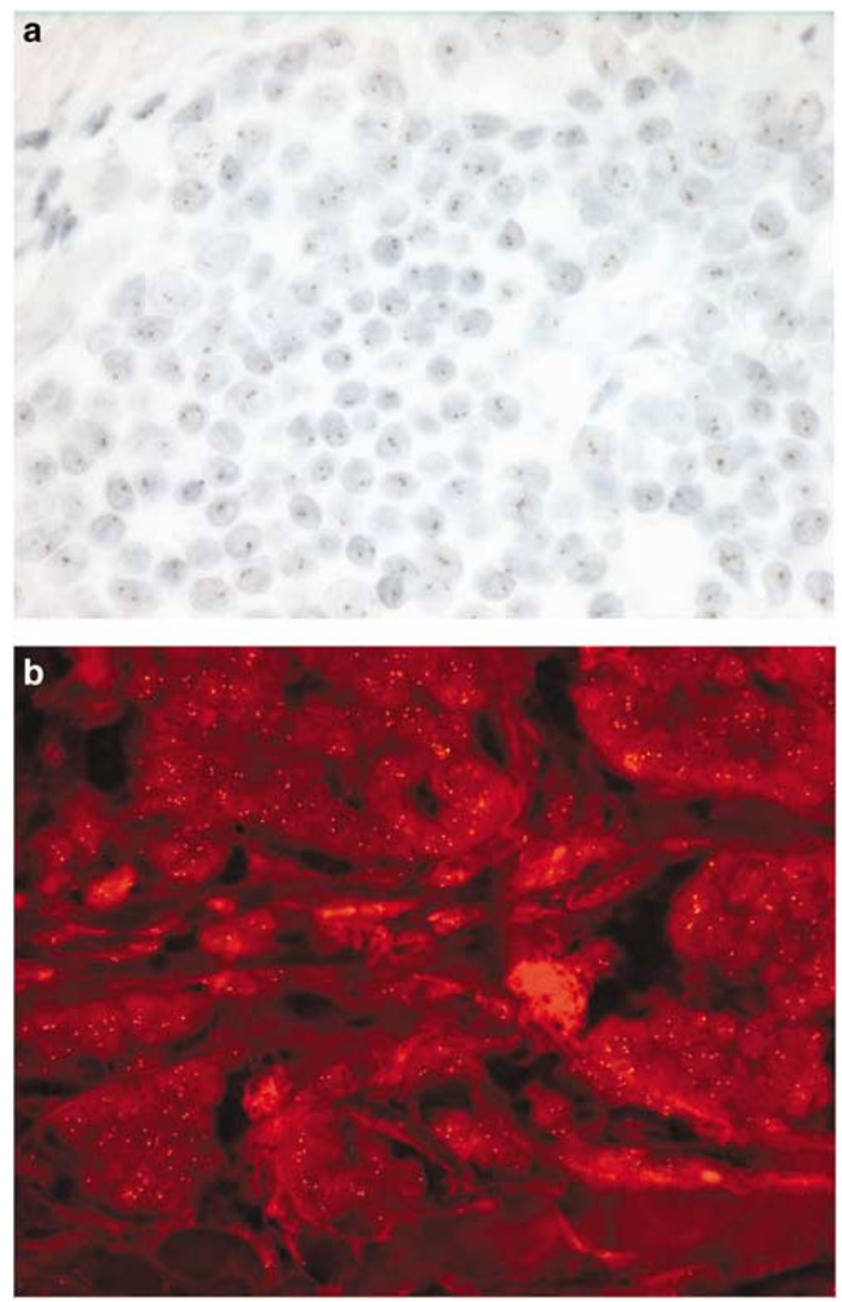

Figure $1 \mathrm{HER} 2 / \mathrm{neu}$ gene not amplified. Immunohistochemistry 0 not shown, concordant (a) CISH (not amplified); (b) FISH (not amplified).

three cases were not amplified by FISH or CISH, suggesting that the immunohistochemistry results for these samples were false-positive. Concordance between immunohistochemistry and FISH/CISH was high. Tumor specimens showing amplification by immunohistochemistry, FISH and CISH are shown in Figure 2. Concordance/discordance results between FISH and CISH are summarized in Table 2. Two CISH negative cases in Group 3 were scored 5 by CISH and are considered borderline negative. It should be noted however that, subsequent to this study being concluded, the Zymed scoring system for CISH amplification was introduced and a score of $>5$ is considered positive, meaning that these two cases would now be classed as CISH positive. The kappa coefficient comparing FISH with CISH was calculated as 0.9131 , indicating an excellent degree of concordance between FISH and CISH. The overall concordance in the three groups was $242 / 254$ or $95.3 \%$, with the great majority of discordant cases (11/12) occurring in samples with very low or borderline amplification with FISH. Examples of different CISH patterns of HER2 oncogene expression are shown in Figure 3. From this study, we propose to add CISH as an alternative to FISH in the HER2 testing algorithm (Figure 4).

\section{Discussion}

There exists a real clinical need for a rapid and accurate test of HER2 status in breast cancer patients. Accurate assessment of HER2 status is essential for identifying patients with cancer who are candidates for treatment with the anti-HER2 monoclonal antibody trastuzumab, which has been shown to significantly prolong survival in HER2positive breast cancer. ${ }^{5,6}$ Response to some types of chemotherapy and hormonal therapy also appears to be dependent on HER2 status. ${ }^{9-14}$ Results of this study show CISH to be a practical and reliable alternative to FISH for the assessment of HER2 gene amplification in breast cancer specimens. A very high level of concordance was seen between CISH and FISH in each of the tumor sample groups tested. This ranged from $93 \%$ in equivocal immunohistochemistry samples to $98 \%$ in HER2 immunohistochemistry-positive cases and $97 \%$ in HER2 immunohistochemistry-negative cases, with an overall concordance rate between CISH and FISH of $>95 \%$. These findings are consistent with the results of previous studies, which have shown concordance rates between CISH and FISH and/or immunohistochemistry to be in the range of 90$100 \% .{ }^{15-21} \mathrm{~A}$ recent study by Gong et $a l^{21}$ evaluated the HER2 status of 80 archival invasive breast carcinoma specimens by CISH and FISH, and also assessed the interobserver reproducibility of CISH results between three pathologists. A 91\% agreement in CISH results between pathologists was reported and all these cases had HER2 status matching the corresponding FISH results. The 

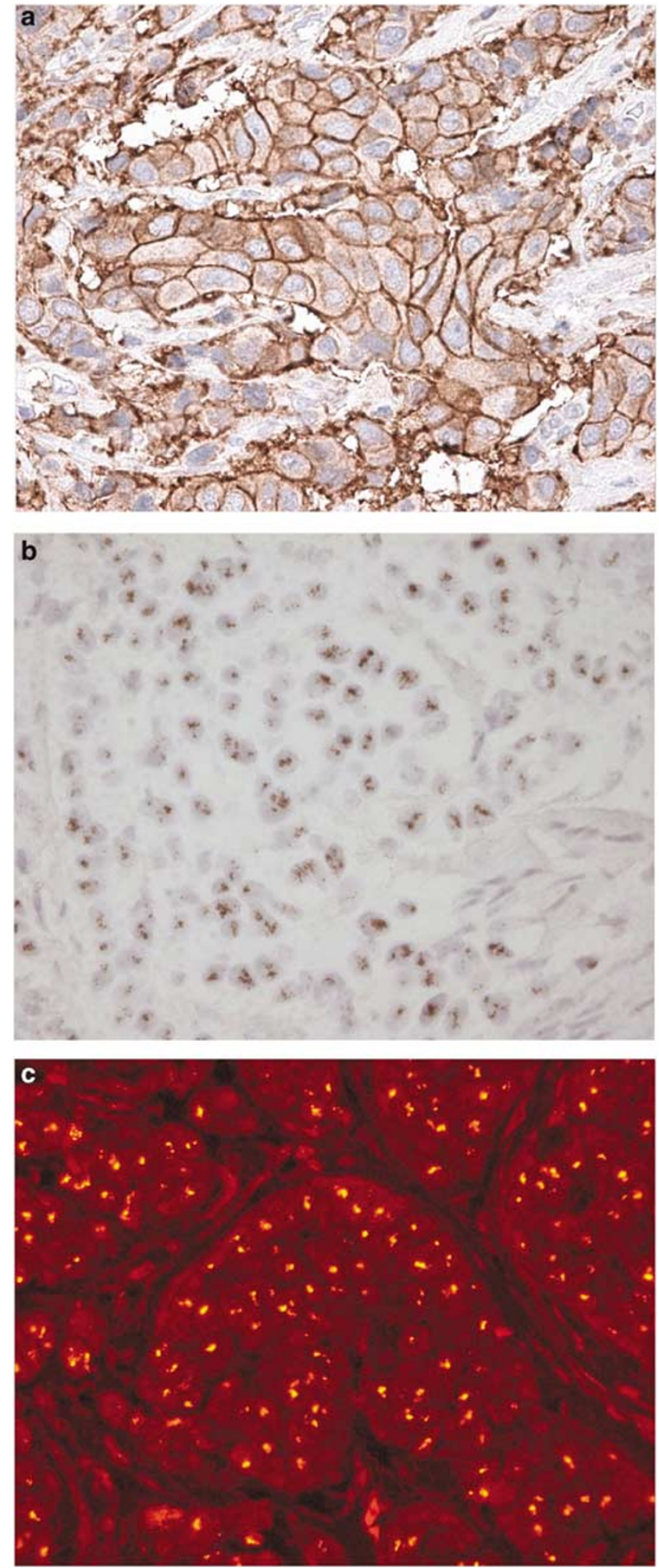

Figure $2 \mathrm{HER} 2 / \mathrm{neu}$ gene amplified showing (a) IHC $3+$ and multiple clusters with (b) CISH and (c) FISH.

overall concordance between CISH and FISH was 0.96, 0.95 and 0.94 for each of the three pathologists, respectively. Discordant CISH results were reported in a small number of cases (7/80), and when these results were compared to the corresponding FISH results, major discrepancies in HER2 status were found in two, three and four cases, respectively. Our study involved evaluation of 254 specimens and included a greater number of problematic borderline or equivocal cases (135), as determined by immunohistochemistry.

A total of 12 discordant cases were identified during this study. Considering the FISH-negative/ CISH-positive discordant cases, possible explanations include low-level polysomy or perhaps erroneous counting of nonspecific granular deposits on the nuclei in cases with low-level amplification, although this latter explanation is less likely. However, if significant granular deposits are observed, particularly outside the nuclei, then the test should be repeated. The cases that were CISH negative but FISH positive could be the result of either excessive digestion in the CISH assay associated with poor fixation, or clumping of the granular signal, which can sometimes occur with overlapping nuclei, resulting in counting two signals as one.

The kappa coefficient for the present study was calculated as 0.91. This compares very favorably with previously reported values comparing FISH and CISH. Bilous et $a l^{18}$ reported kappa coefficients of 1.0, 0.88 and 0.5 , while Tanner et $a l^{15}$ reported a kappa coefficient of 0.81 for their study and Park et $a l^{22}$ calculated a kappa coefficient of 0.84 .

The typical pattern of HER2 gene amplification by CISH is a peroxidase-positive cluster of multiple gene copies or individual scattered signals, both of which are easily identified using $\times 25$ or $\times 40$ objective magnification. The most common interpretation difficulties encountered in this study were possible overestimation of the number of gene copies per nucleus due to overlapping nuclei in thick sample areas and occasional scattered particulate debris on slides, which may have obscured the peroxidase-positive dots. Very occasionally, slight differences in the average number of gene copies per nucleus were seen between areas of invasive and in-situ carcinoma. In strongly positive cells, the number of gene copies per nucleus may have been underestimated due to overlapping dots. This problem of coalescing signal clusters in areas of high-level amplification with CISH has been highlighted previously. ${ }^{15,16}$ However, a recent international ring study has shown CISH results to be highly reproducible between laboratories. ${ }^{23}$ With CISH, precise enumeration of gene copies exceeding 10 is not required for routine diagnostics. The most important category in CISH is low-level amplification, which does require determination of the precise number of gene copies per nucleus for accurate assessment of HER2 gene amplification status.

As in previous studies, the great majority of discordant cases in the present study occurred in samples with very low-level amplification with 
Table 2 Concordance/discordance between FISH and CISH

\begin{tabular}{|c|c|c|c|c|c|c|c|c|}
\hline \multirow[t]{3}{*}{ Group } & \multirow[t]{3}{*}{ Immunohistochemistry result } & & & \multicolumn{5}{|c|}{ FISH/CISH discordance } \\
\hline & & \multicolumn{2}{|c|}{ FISH/CISH concordance } & \multicolumn{3}{|c|}{ FISH } & \multicolumn{2}{|c|}{$\mathrm{CISH}$} \\
\hline & & Number & $\%$ & Number & Positive & Negative & Positive & Negative \\
\hline 1 & $0 / 1+(n=69)$ & $67 / 69$ & 97 & $2 / 69$ & - & 2 & 2 & - \\
\hline 2 & $3+(n=50)$ & $49 / 50$ & 98 & $1 / 50$ & - & 1 & 1 & - \\
\hline \multirow[t]{2}{*}{3} & $2+(n=135)$ & $126 / 135$ & 93 & $9 / 135$ & 6 & 3 & 1 & 8 \\
\hline & Total & $242 / 254$ & 95.3 & 12 & 6 & 6 & 4 & 8 \\
\hline
\end{tabular}
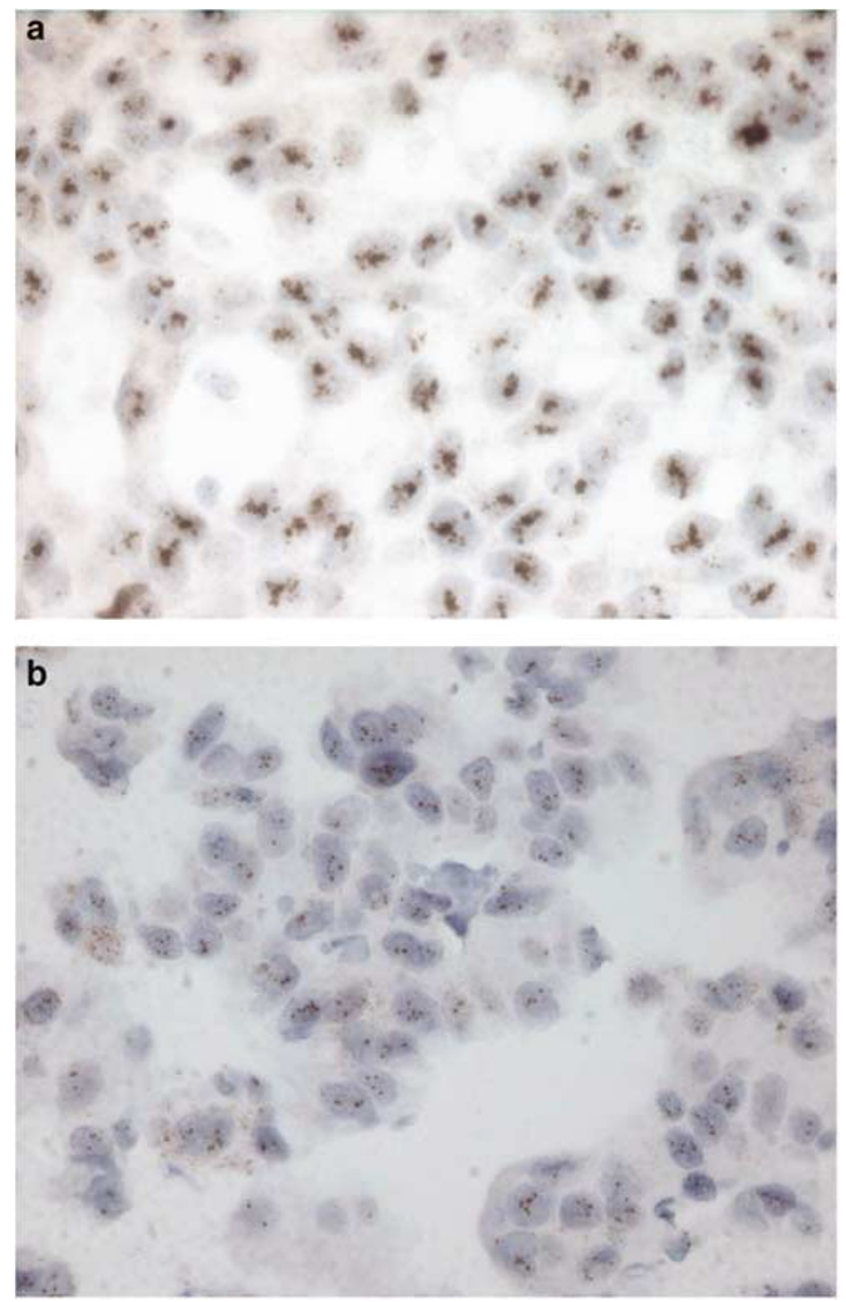

Figure 3 Different patterns of CISH amplification: (a) clusters; (b) single signals.

FISH. This being the case, it may be advisable to either run parallel sections to stain for chromosome 17 centromere to rule out the possibility of polysomy, repeat the test if there is nonspecific granularity on the nuclei and cytoplasm, or possibly modify the digestion time in cases that are poorly fixed in order to avoid both false-positive and false-negative results by CISH. The number of discordant cases in

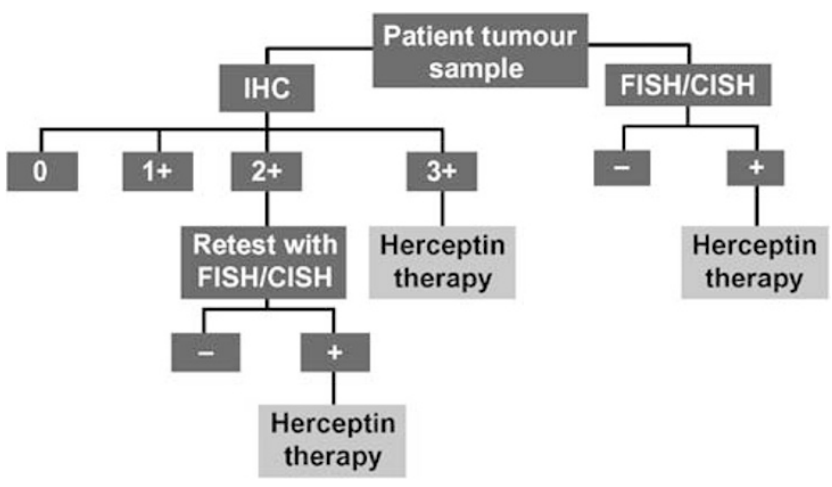

Figure 4 The recommended HER2/neu testing algorithm.

the study accounts for less than $5 \%$ of the total (12/ 254 ) and these have a very low level of amplification by FISH. The clinical benefit of trastuzumab therapy in this group is questionable; however, additional clinical validation data is required to confirm that this is the case. Accurate scoring of samples with low-level FISH amplification is known to be technically challenging, and interobserver variability is greatest in this category of samples. According to FISH scoring guidelines, these cases are positive for HER2 amplification. However, results of another recent study found that $43 \%$ of all cases scored as having a low level of amplification by FISH were nonamplified by CISH. ${ }^{20}$ The nature of the two tests may contribute to the discrepancy between CISH and FISH in cases of borderline amplification. With FISH, both the number of copies of HER2 and CEP17 must be counted to yield the final score. In contrast, CISH scores are based only on assessment of the number of HER2 copies. Although CISH does not routinely use an intrinsic marker for chromosome 17 centromere, there is a new, commercially available and validated Zymed probe that is easy to use. Although this probe does not target the same section as the probe for the HER2/neu gene, it targets an adjacent section. This could be a useful tool to rule out the possibility of polysomy, particularly in cases with low-level amplification. However, polysomy does not appear to be a significant problem in tumors with high levels of HER2/neu gene 
amplification. ${ }^{24}$ While the clinical significance of such discrepancies between CISH and FISH for the scoring of samples with borderline amplification remains to be fully determined and clinically validated, it should be noted that only $1-3 \%$ of all breast cancers fall into this category in clinical practice ${ }^{23}$ and response to trastuzumab is related to a high level of HER2 expression and amplification.

The advantages of CISH over FISH are manifold. In particular, CISH methodology is more accessible and less costly than FISH. Unlike FISH, CISH does not require fluorescent microscopy equipment or experience in interpreting fluorescent signals. Interpretation of CISH results requires only standard laboratory equipment and is generally quick and easy. In addition, CISH signal intensity does not diminish over time. This allows CISH slides to be stored in standard slide archives at room temperature for future reference, eliminating the need to photograph results for documentation. Furthermore, while assessment of tumor morphology is suboptimal with FISH, the hematoxylin-stained CISH slides allow for easy correlation and/or verification with histopathological findings, which would be expected to result in a lower sampling error. This study suggests the use of CISH as an alternative to FISH, allowing pathologists who have no access to costly fluorescence microscopy the ability to assess their equivocal cases by in-situ hybridization. However, the primary testing method for HER2/ neu will remain the same, and only equivocal cases will require confirmation by in-situ hybridization methods, which could be FISH or CISH. Although the cost per case is lower for CISH (Vysis PathVysion HER2 FISH kit (20 tests), CAD\$2650.00 (CAD\$132.50 per test) and Zymed HER2 CISH kit (20 tests), CAD $\$ 1442.00$ (CAD\$72.10 per test)), the real issue is the other advantages of CISH over FISH, such as comparative ease of use and the ability to store samples without degradation. It is important to emphasize that any new methodology requires a vigorous quality assurance program for any laboratory to adopt it as their method of choice.

In summary, the results of this study suggest that CISH is well-suited for use as an alternative to FISH in the HER2 testing algorithm. Compared with FISH, CISH offers a convenient method for the assessment of HER2 gene amplification status in routinely processed immunohistochemistry equivocal breast cancer samples, using equipment widely available in diagnostic pathology laboratories.

\section{Acknowledgements}

We thank F Hoffmann-La Roche Ltd for providing funding for the preparation of this manuscript and Jennifer Coward and Karen Runcie for editorial support.

\section{References}

1 Owens MA, Horten BC, Da Silva MM. HER2 amplification ratios by fluorescence in situ hybridization and correlation with immunohistochemistry in a cohort of 6556 breast cancer tissues. Clin Breast Cancer 2004;5:63-69.

2 Ross JS, Fletcher JA, Bloom KJ, et al. Targeted therapy in breast cancer: the HER-2/neu gene and protein. Mol Cell Proteomics 2004;3:379-398.

3 Slamon DJ, Clark GM, Wong SG, et al. Human breast cancer: correlation of relapse and survival with amplification of the HER-2/neu oncogene. Science 1987;235:177-182.

4 Slamon DJ, Godolphin W, Jones LA, et al. Studies of the HER-2/neu proto-oncogene in human breast and ovarian cancer. Science 1989;244:707-712.

5 Slamon DJ, Leyland-Jones B, Shak S, et al. Use of chemotherapy plus a monoclonal antibody against HER2 for metastatic breast cancer that overexpresses HER2. N Engl J Med 2001;344:783-792.

6 Extra J-M, Cognetti F, Maraninchi D, et al. Trastuzumab (Herceptin ${ }^{\mathbb{R}}$ ) plus docetaxel vs docetaxel alone as firstline treatment of HER2-positive metastatic breast cancer (MBC): results of a randomised multicentre trial (abstract 239). Eur J Cancer 2004;2(Suppl 3):125.

7 van de Vijver M. Emerging technologies for HER2 testing. Oncology 2002;63(Suppl 1):33-38.

8 Bilous M, Dowsett M, Hanna W, et al. Current perspectives on HER2 testing: a review of national testing guidelines. Mod Pathol 2003;16:173-182.

9 Leitzel K, Teramoto Y, Konrad K, et al. Elevated serum c-erbB-2 antigen levels and decreased response to hormone therapy of breast cancer. J Clin Oncol 1995; 13:1129-1135.

10 Budman DR, Berry DA, Cirrincione CT, et al. Dose and dose intensity as determinants of outcome in the adjuvant treatment of breast cancer. The Cancer and Leukemia Group B. J Natl Cancer Inst 1998;90:1205-1211.

11 Hanna W. Testing for HER2 status. Oncology 2001;61(Suppl 2):22-30.

12 Lebeau A, Deimling D, Kaltz C, et al. HER-2/neu analysis in archival tissue samples of human breast cancer: comparison of immunohistochemistry and fluorescence in situ hybridization. J Clin Oncol 2001; 19:354-363.

13 Piccart M, Lohrisch C, Di Leo A, et al. The predictive value of HER2 in breast cancer. Oncology 2001; 61(Suppl 2):73-82.

14 de Laurentiis M, Arpino G, Massarelli E, et al. HER2 as predictive marker of resistance to endocrine treatment (ET) for advanced breast cancer (ABC): a meta-analysis of published studies (abstract 233). Breast Cancer Res Treat 2002;76(Suppl 1):S68.

15 Tanner M, Gancberg D, Di Leo A, et al. Chromogenic in situ hybridization: a practical alternative for fluorescence in situ hybridization to detect HER-2/neu oncogene amplification in archival breast cancer samples. Am J Pathol 2000;157:1467-1472.

16 Dandachi N, Dietze O, Hauser-Kronberger C. Chromogenic in situ hybridization: a novel approach to a practical and sensitive method for the detection of HER2 oncogene in archival human breast carcinoma. Lab Invest 2002;82:1007-1014.

17 Zhao J, Wu R, Au A, et al. Determination of HER2 gene amplification by chromogenic in situ hybridization (CISH) in archival breast carcinoma. Mod Pathol 2002;15:657-665. 
18 Bilous M, Morey A, Armes J, et al. CISH or FISH? The interlaboratory reproducibility of CISH testing for HER2 and correlation with IHC and FISH results (abstract 160). Eur J Cancer Suppl 2004;2:100.

19 Peiro G, Mayr D, Hillemanns P, et al. Analysis of HER2/neu amplification in endometrial carcinoma by chromogenic in situ hybridization. Correlation with fluorescence in situ hybridization, HER-2/neu, p53 and Ki-67 protein expression, and outcome. Mod Pathol 2004;17:227-287.

20 van de Vijver MJ, Bilous M, Hanna W, et al. Correlation of chromogenic in-situ hybridisation (CISH) with FISH and IHC for assessment of HER2 gene amplification: an international validation ring study (abstract 157). Eur J Cancer Suppl 2004;2:99.

21 Gong Y, Gilcrease M, Sneige N. Reliability of chromogenic in situ hybridization for detecting HER-2 gene status in breast cancer: comparison with fluorescence in situ hybridization and assessment of interobserver reproducibility. Mod Pathol 2005;18:1015-1021.

22 Park K, Kim J, Lim S, et al. Comparing fluorescence in situ hybridization and chromogenic in situ hybridization methods to determine the HER2/neu status in primary breast carcinoma using tissue microarray. Mod Pathol 2003;16:937-943.

23 van de Vijver M, Bilous M, Hanna W, et al. Chromogenic in-situ hybridisation (CISH) compared with FISH and IHC for detection of HER2 gene amplification: an international validation ring study. Breast Cancer Res Treat 2003;82(Suppl 1) (abstract 324) S75.

24 Varshney D, Zhou YY, Geller SA, et al. Determination of HER2 status and chromosome 17 polysomy in breast carcinomas comparing HercepTest and PathVysion FISH assay. Am J Clin Pathol 2004;121:70-77. 